\title{
Mapping evidence on women's knowledge and practice of breast self-examination in sub-Saharan Africa: a scoping review protocol
}

\author{
Roseline H. Udoh', Monica Ansu-Mensah', Mohammed Tahiru', Vitalis Bawontuo ${ }^{1,3}$ and Desmond Kuupiel ${ }^{2,3^{*}}$ [D
}

\begin{abstract}
Background: Globally, breast cancer is the most common malignant condition in women. Breast self-examination practice following correct procedure potentially can lead to early detection of breast abnormalities. We propose to systematically chart literature and examine the scope of evidence on women's knowledge and practice of breast self-examination in sub-Saharan Africa (SSA).

Methods: Our scoping review methods will be guided by the framework proposed by Arksey and O'Malley, Levac et al. and Joanna Briggs Institute guidelines. Literature searches will be conducted in the following electronic databases (from 2008 onwards): PubMed/MEDLINE, Scopus, Web of Science, CINAHL, PsycINFO and Health Sources. Grey literature will be identified through searching dissertation databases, Google Scholar and governmental databases. Two reviewers will screen all citations and full-text articles We will abstract data, organise them into themes and sub-themes, summarise them and report the results using a narrative synthesis. The study methodological quality (or bias) will be appraised using a mixedmethod appraisal tool.

Discussion: The findings from the scoping review will contribute to obtain an understanding of the women's knowledge and practice of breast self-examination in sub-Saharan Africa, and will likely reveal the depth of evidence helping to identify gaps for future research. Results will be published in a peer-reviewed journal. Implications for clinical practice and health policy will be discussed.
\end{abstract}

Keywords: Women, Breast cancer, Breast self-examination, Knowledge, Practice, Early detection, and Sub-Saharan Africa

\section{Background}

Breast cancer $(\mathrm{BC})$ is the most common malignant condition in women and the second most common malignant condition worldwide [1], and the leading cause of cancerrelated deaths among women [2, 3]. In 2012, BC alone accounted for 1.7 million (12.1\%) of the total 14.1 million women diagnosed with cancer worldwide [3-5]. Of the $12.1 \%$ of women diagnosed with BC, globally in 2012, about $56.8 \%$ were diagnosed in low- and middle-income countries (LMICs) [4]. Over 522,000 BC deaths among women were recorded in the same year, and the majority

\footnotetext{
* Correspondence: desmondkuupiel98@hotmail.com

${ }^{2}$ Department of Public Health Medicine, School of Nursing and Public Health, University of KwaZulu-Natal, Durban, South Africa

${ }^{3}$ Research for Sustainable Development Consult, Sunyani, Ghana

Full list of author information is available at the end of the article
}

of these deaths occurred in sub-Saharan Africa (SSA) [6, 7]. Global projections on BC burden indicates that over 19 million new cases will occur by the year 2020 and 2025 [6, $8-11$ ]. Of these 19.7 million $\mathrm{BC}$ new cases, 10.6 million cases will occur in LMICs, accounting for over 1 million $\mathrm{BC}$ new cases per year [5, 8-10]. At the same time, global $\mathrm{BC}$ mortality projections also show that 8.5 million women will die, of which 3.9 million of these deaths will occur in LMICs causing more than 1.5 million premature and preventable deaths $[4,8]$.

These projections on $\mathrm{BC}$ morbidity and mortality are worrying and require global actions to prevent $\mathrm{BC}$ and improve the health outcomes of $\mathrm{BC}$ patients. To enable early detection of $\mathrm{BC}$ and prompt linkage to care, screening examination of the breast such as breast self-examination

(c) The Author(s). 2020 Open Access This article is distributed under the terms of the Creative Commons Attribution 4.0 International License (http://creativecommons.org/licenses/by/4.0/), which permits unrestricted use, distribution, and reproduction in any medium, provided you give appropriate credit to the original author(s) and the source, provide a link to the Creative Commons license, and indicate if changes were made. The Creative Commons Public Domain Dedication waiver (http://creativecommons.org/publicdomain/zero/1.0/) applies to the data made available in this article, unless otherwise stated. 
(BSE) and/or clinical breast examination (CBE) are highly essential. Regular screening of the breast facilitates recognition of breast abnormalities such as the lesion or lump as early as possible and link patients to various treatment options and supportive management [2, 12, 13]. It has been reported that there are more treatment choices for $\mathrm{BC}$ patients and a better chance for survival associated with early $\mathrm{BC}$ detected [2]. A survival rate of approximately $93 \%$ or more has been reported for women whose $\mathrm{BC}$ is detected at an early stage (first 5 years) [2]. For early detection of breast lesion or lump, an examination of the breast should be done either by the individual BSE or by health personnel CBE $[14,15]$. However, due to several reasons such as poor health-seeking behaviour, poor access to healthcare, late reporting of $\mathrm{BC}$ to health facilities and limited availability of cancer diagnostic and treatment facilities, particularly in SSA, the concept of BSE and reporting of breast abnormalities for CBE is vital. Knowledge and practice of BSE following the correct procedure has been demonstrated to be essential for self-detection of any breast abnormalities and reporting to health facilities for prompt initiation of clinical interventions such as $\mathrm{CBE}$, diagnosis and treatment [12, 14, 15]. This proposed scoping review will, therefore, aim to chart literature and examine the scope of evidence on the knowledge and practice of BSE among women in SSA.

\section{Methods}

\section{Overview}

Scoping review maps a range of literature existing around a research field of interest to identify gaps for future research $[16,17]$. A scoping review is also helpful in determining the need and value of a primary study or systematic review [16]. This study will be guided by the 2005 Arksey and O'Malley scoping review framework incorporating the Levac et al. 2010 recommendation [16, 18] which stipulates the following: identification of the research questions; identification of relevant studies; study selection; charting and collating of data, summarising and reporting findings, and the 2015 Joanna Briggs Institute guidelines [19]. The present protocol is being reported in accordance with the reporting guidance provided in the Preferred Reporting Items for Systematic Reviews and Meta-Analyses Protocol (PRISMA-P) statement [20] (see checklist in Additional file 1).

\section{Identifying the research question}

Our overall research question will be: What are the evidence on women's knowledge and practice of BSE in SSA?

The sub-review questions will be as follows:

- What evidence exists on the knowledge of BSE among women in SSA?
- What evidence exists on the practice of BSE among women in SSA?

- Is there evidence linking early BC detection among women to BSE in SSA?

\section{Information sources and search strategy}

A comprehensive search for relevant primary studies published in peer-reviewed journals on women's knowledge and practice of BSE will be done in the following databases (from 2008 onwards): PubMed, SCOPUS, Web of Science, Google Scholar and EBSCOhost (Academic Search Complete, MEDLINE, CINAHL, PsycINFO and Health Sources) using a combination of appropriate keywords and index terms. The keywords for the online database search will include "women" OR "woman" OR "female" OR "mothers" AND "breast" OR "Breast" OR "breast cancer" OR "cancer" OR"carcinoma" OR "lumps" OR "tumour"OR "neoplasms" OR "tumor" OR "malignancy" OR "benign" AND "breast self-examination" OR "self-breast examination", OR "examination" OR "screening" AND "knowledge" OR "practice" OR "early detection" OR "diagnosis", "sub-saharan africa" OR "Africa". We will also search by country names to enable us to access all relevant articles. Medical Subject Heading $(\mathrm{MeSH})$ terms in all fields will also be included to enable the identification of all relevant studies. Limitations on study design, date of publication and language will be removed during the search for potential articles in the databases. Relevant grey literature like unpublished studies, thesis and dissertations will also be included. We will additionally search for relevant literature from the World Health Organization and governmental websites, and from the reference list of included articles. A detailed searched record will be documented as follows: date of search, search engine, keywords and number of retrieved publications and the number of eligible studies. A draft search strategy for PubMed/MEDLINE is provided in Additional file 2.

\section{Eligibility criteria}

To be included in the scoping review, an article will have to measure or focus on knowledge and practice of BSE in the conceptual framework. Peer-reviewed journal articles and unpublished reports will be included if they are published between the period of 2008-to date of final search, are written in English, involved adult women participants (18 years and above), described evidence in the context of early detection of BC with BSE and are conducted in SSA countries. Quantitative, qualitative and mixed-method studies will be included in order to consider different aspects of measuring knowledge and practices. Papers will be excluded if they did not fit into the conceptual framework of the study, focused on men, $\mathrm{CBE}, \mathrm{BC}$ detection using mammography or treatment of BC. In addition, reviews will be excluded. 


\section{Selection of sources of evidence}

A comprehensive title screening will be done by the principal investigator and all eligibility studies imported to endnotes X7 library created for the study. The library will then be cleaned by thoroughly examining and deleting duplicate articles. Following this process, the final endnote library will be shared with co-reviewers for the next stage of the screening process. Two independent reviewers will perform both abstract and full-text screening using the eligibility criteria. Disagreements between reviewers' responses at the abstract screening stage will be addressed via deliberations with the co-reviewer to reach a consensus. Also, disagreements at the full-text screening stage between reviewers for this proposed scoping review will be addressed by consulting a third reviewer. We will also calculate the inter-rater agreement (Cohen's kappa co-efficient $(k)$ statistics) between reviewers' responses as well as McNemar's chi-square statistic. In the case where a full-text article cannot be retrieved or is not accessible from the online databases, we will seek assistance from the Catholic University College library, University of Ghana library or the University of KwaZulu-Natal library. We will also write to the authors to request full-text articles not accessible online. A flow chart showing details of studies included and excluded at each stage of the study selection process will be provided [21].

\section{Charting the data}

We will extract relevant information from the included studies to enable us to answer the proposed scoping review question. To ensure accuracy and consistency in the data that will be extracted, two reviewers will independently pilot the data extraction form using $10 \%$ of the included studies in parallel. The data extraction form will be adjusted before its final use based on feedback from the reviewers. We will also keep the data extraction form updated until all relevant information has been extracted from the included studies. Table 1 illustrates the data extraction form that will be used for the proposed scoping review.

\section{Collating, summarising, and reporting the results}

We will present information about the included articles that align to the objective and questions of the review. We will extract all relevant data from the eligible studies manually using the piloted data extraction form designed in a tabular format to answer the review question. We will also organise all the data extracted into themes and sub-themes, summarise them and report the results using a narrative approach, tables and appropriate figures. Other emerging themes will also be reported. The PRISMA extension for scoping review checklist will be used to guide the result paper of this proposed study.
Table 1 Data extraction form

Author and date of publication

Study title

Study aim/objective

Type of study design

Study setting (country)

Geography setting (rural/urban)

Study population

Number of study participants

Study findings

Significant findings

Conclusions

Subsequently, a systematic review and meta-analysis may be conducted using quantitative data if warranted.

\section{Quality appraisal}

The methodological quality of all included studies will be assessed using the Mixed Method Quality Appraisal Tool (MMAT) version 2018. The included studies will be appraised by reading thoroughly each included article and responding appropriately to the questions for the type of study design as prescribed by the MMAT. To avoid bias, two reviewers will independently perform the quality assessment, and in case of any disagreement, a third reviewer will be contacted to resolve the discrepancies. The score of each appraised article will be calculated and the results interpreted as $\leq 50 \%$ low quality, $51-75 \%$ average quality and $76-100 \%$ high quality [22].

\section{Discussion}

This scoping review is to map literature and examine the scope of evidence on the knowledge and practice of BSE among women in SSA. There has been a rise in morbidity and mortality due to $\mathrm{BC}$ in recent times [12, $23,24]$. Late reporting or detection of $B C$ is seen as contributing to high mortality in SSA [12]. Practicing BSE by women can potentially help early $\mathrm{BC}$ detection at home prior to $\mathrm{CBE}$, diagnosis and prompt initiation of treatment. Therefore, literature published from 2008 to the last search date in 2019 will be searched and screened and the included articles synthesised to enable retrieval of current information on women's knowledge and practice of BSE in SSA. This will also enable us to know the extent of recent (from 2008 to 2019) research activity on BSE among women in SSA and identify gaps for recommendations on future research. Due to the lack of expertise for other languages such as French, Spanish, German, Arabic and others, we will limit the publication language to only English during the abstract and fulltext screening stages. Notwithstanding this, we anticipate retrieving many articles for this review. Although 
the inclusion of methodology appraisal of included articles is not mandatory for a scoping review study, we will include it to help report on the risk of bias and to inform the quality of the evidence.

The implications, strengths and limitations will be adequately reported. We anticipate that the findings from this proposed scoping review will help improve BSE among women by key stakeholders, reveal research gaps to inform future research to provide evidence-based solutions to improve BSE among women, and contribute to early $\mathrm{BC}$ detection, diagnosis and treatment of $\mathrm{BC}$ in SSA. The finding of this proposed study may also inspire countries in SSA to advocate for less-expensive and simple diagnostic tests for use in resource-limited settings or self-testing to facilitate early detection of $\mathrm{BC}$ at primary healthcare clinics and by individuals at homes to reduce the impact $\mathrm{BC}$ in SSA.

\section{Supplementary information}

Supplementary information accompanies this paper at https://doi.org/10. 1186/s13643-019-1254-7.

Additional file 1. PRISMA-P 2015 Checklist.

Additional file 2. Pilot search in PubMed electronic database.

\section{Abbreviations}

BC: Breast cancer; BSE: Breast self-examination; LMIC: Low- and middleincome countries; SSA: Sub-Saharan Africa

\section{Acknowledgements}

We are grateful to the Catholic University College of Ghana, University of KwaZulu-Natal and Handmaids of the Holy Child Jesus congregation (Ghana Province) for making available the necessary resources for completion of this study protocol.

\section{Authors' contributions}

RHU conceptualised the study under the supervision of DK. RHU wrote the first draft. MA-M and MT contributed to the writing of the protocol. VB and DK made critical revisions. All authors approved the final draft.

\section{Funding}

No funding has been obtained yet for this proposed study. However, we are still soliciting for funding from The Handmaids of the Holy Child Jesus congregation (Ghana Province).

\section{Availability of data and materials}

We have duly cited all studies, and data is presented in the form of references.

\section{Ethics approval and consent to participate}

Not applicable

\section{Consent for publication}

Not applicable

\section{Competing interests}

The authors declare that they have no competing interests.

\section{Author details}

${ }^{1}$ Faculty of Health and Allied Sciences, Catholic University College of Ghana, Fiapre, Sunyani, Ghana. ${ }^{2}$ Department of Public Health Medicine, School of Nursing and Public Health, University of KwaZulu-Natal, Durban, South Africa. ${ }^{3}$ Research for Sustainable Development Consult, Sunyani, Ghana.
Received: 16 May 2019 Accepted: 11 December 2019

Published online: 06 January 2020

\section{References}

1. Rivera-Franco MM, Leon-Rodriguez E. Delays in breast cancer detection and treatment in developing countries. Breast Cancer. 2018;12: 1178223417752677

2. Milgard C. Breast Center USA2019 [cited 2019 8/4/2019]. Available from: http://www.carolmilgardbreastcenter.org/early-detection.

3. Angaher. An overview of breast cancer epidemiology, risk factors, pathophysiology and cancer risk reduction. 2017;1:00019.

4. Are C, Rajaram S, Are M, Raj H, Anderson BO, Chaluvarya Swamy R, et al. A review of global cancer burden: trends, challenges, strategies, and a role for surgeons. 2013;107(2):221-6.

5. Anderson BO. Breast cancer-thinking globally: American Association for the Advancement of Science; 2014

6. Fletcher C, Bridge J, Hogendoorn P, Mertens FJWHO. WHO classification of tumours of soft tissue and bone (IARC WHO classification of tumours); 2013.

7. Sinn H-P, Kreipe HJBC. A brief overview of the WHO classification of breast tumors. 2013;8(2):149-54

8. Gradishar WJ, Anderson BO, Blair SL, Burstein HJ, Cyr A, Elias AD, et al. Breast cancer version 3.2014. J Natl Compr Canc Netw 2014;12(4):542-590.

9. Distelhorst SR, Cleary JF, Ganz PA, Bese N, Camacho-Rodriguez R, Cardoso F, et al. Optimisation of the continuum of supportive and palliative care for patients with breast cancer in low-income and middle-income countries: executive summary of the Breast Health Global Initiative, 2014. Lancet Oncol. 2015;16(3):e137-e47.

10. Ferlay J, Soerjomataram I, Ervik M, Dikshit R, Eser S, Mathers C, et al. GLOBOCAN 2012 v1. 0, Cancer incidence and mortality worldwide: IARC CancerBase No. 11. 2013. 2014.

11. Cumber SN, Nchanji KN, Tsoka-Gwegweni JMJSAJoGO. Breast cancer among women in sub-Saharan Africa: prevalence and a situational analysis. 2017;9(2):35-37.

12. Black E, Richmond R. Improving early detection of breast cancer in subSaharan Africa: why mammography may not be the way forward. Global Health. 2019;15(1):3.

13. Hassan LM, Mahmoud N, Miller AB, Iraj H, Mohsen M, Majid J, et al. Evaluation of effect of self-examination and physical examination on breast cancer. Breast. 2015;24(4):487-90.

14. Yip CH, Smith RA, Anderson BO, Miller AB, Thomas DB, Ang ES, et al. Guideline implementation for breast healthcare in low-and middle-income countries: Early detection resource allocation. 2008;113(\$8):2244-56.

15. Echavarria Ml, Anderson BO, Duggan C, Thompson B. Global uptake of BHGl guidelines for breast cancer. Lancet Oncol. 2014;15(13):1421-3.

16. Arksey H, O'Malley L. Scoping studies: towards a methodological framework. Int J Soc Res Methodol. 2005;8(1):19-32.

17. Peters MD, Godfrey CM, Khalil H, Mclnerney P, Parker D, Soares CB. Guidance for conducting systematic scoping reviews. Int J Evid Based Healthc. 2015:13(3):141-6.

18. Levac D, Colquhoun H, O'Brien KK. Scoping studies: advancing the methodology. Implement Sci. 2010:5(1):69.

19. Institute JB. Joanna Briggs Institute reviewers' manual: 2015 edition/ supplement: Methodology for JBI Scoping Reviews Adelaide: The Joanna Briggs Institute; 2015.

20. Moher D, Shamseer L, Clarke M, Ghersi D, Liberati A, Petticrew M, et al. Preferred reporting items for systematic review and meta-analysis protocols (PRISMA-P) 2015 statement. Syst Rev. 2015;4(1) https//doi.org/10.1186/2046-4053-4-1.

21. Moher D, Liberati A, Tetzlaff J, Altman DG, The PRISMA Group. PRISMA 2009 Flow Diagram. 2009;6(2009):1000097.

22. Kuupiel D, Bawontuo V, Mashamba-Thompson TP. Mapping evidence on tuberculosis active case finding policies, strategies, and interventions for tuberculosis key populations: a. systematic scoping review protocol. Systematic Reviews. 2019;8(1):162.

23. Kisiangani J, Baliddawa J, Marinda P, Mabeya H, Choge JK, Adino EO, et al. Determinants of breast cancer early detection for cues to expanded control and care: the lived experiences among women from Western Kenya. BMC Womens Health. 2018;18(1):81.

24. Braun V, Clarke V, Hayfield N, Terry GJHoRMiHSS. Thematic analysis. 2019:843-860.

\section{Publisher's Note}

Springer Nature remains neutral with regard to jurisdictional claims in published maps and institutional affiliations. 